\title{
Integrated Multi-Omic Analysis of Mycobacterium tuberculosis H37Ra Redefines Virulence Attributes
}

\section{OPEN ACCESS}

Edited by:

Steve Lindemann,

Purdue University, United States

Reviewed by:

Subramanian Dhandayuthapani, Texas Tech University Health Sciences Center, United States

Henrique César Pereira Figueiredo, Universidade Federal de Minas Gerais,

Brazil

*Correspondence:

Sneha M. Pinto

sneha@yenepoya.edu.in

T. S. Keshava Prasad

keshav@yenepoya.edu.in

†These authors have contributed equally to this work.

$¥$ Present Address: Saketh Kapoor

Stem Cells and Regenerative

Medicine Centre, Yenepoya Research

Centre, Yenepoya (Deemed to be

University), Mangalore, India

Specialty section:

This article was submitted to

Systems Microbiology,

a section of the journal

Frontiers in Microbiology

Received: 23 February 2018 Accepted: 30 May 2018

Published: 19 June 2018

Citation:

Pinto SM, Verma R, Advani J, Chatterjee O, Patil AH, Kapoor S, Subbannayya Y, Raja R, Gandotra S and Prasad TSK (2018) Integrated

Multi-Omic Analysis of

Mycobacterium tuberculosis H37Ra

Redefines Virulence Attributes.

Front. Microbiol. 9:1314.

doi: 10.3389/fmicb.2018.01314

\begin{abstract}
Sneha M. Pinto ${ }^{1 * t}$, Renu Verma ${ }^{2 \dagger}$, Jayshree Advani ${ }^{2,3 \dagger}$, Oishi Chatterjee ${ }^{1,2,4}$, Arun H. Patil ${ }^{1,2,5}$, Saketh Kapoor ${ }^{1 \neq}$, Yashwanth Subbannayya ${ }^{1}$, Remya Raja ${ }^{2}$, Sheetal Gandotra ${ }^{6}$ and T. S. Keshava Prasad ${ }^{1,2 *}$
\end{abstract}

${ }^{1}$ Center for Systems Biology and Molecular Medicine, Yenepoya (Deemed to be University), Mangalore, India, ${ }^{2}$ Institute of Bioinformatics, International Technology Park, Bangalore, India, ${ }^{3}$ Manipal Academy of Higher Education, Manipal, India, ${ }^{4}$ School of Biotechnology, Amrita Vishwa Vidyapeetham, Kollam, India, ${ }^{5}$ School of Biotechnology, KIIT University, Bhubaneswar, India, ${ }^{6}$ CSIR-Institute of Genomics and Integrative Biology, New Delhi, India

$\mathrm{H} 37 \mathrm{Ra}$ is a virulence attenuated strain of Mycobacterium tuberculosis widely employed as a model to investigate virulence mechanisms. Comparative high-throughput studies have earlier correlated its avirulence to the presence of specific mutations or absence of certain proteins. However, a recent sequencing study of H37Ra, has disproved several genomic differences earlier reported to be associated with virulence. This warrants further investigations on the H37Ra proteome as well. In this study, we carried out an integrated analysis of the genome, transcriptome, and proteome of H37Ra. In addition to confirming single nucleotide variations (SNVs) and insertion-deletions that were reported earlier, our study provides novel insights into the mutation spectrum in the promoter regions of 7 genes. We also provide transcriptional and proteomic evidence for 3,900 genes representing $\sim 80 \%$ of the total predicted gene count including 408 proteins that have not been identified previously. We identified 9 genes whose coding potential was hitherto reported to be absent in H37Ra. These include 2 putative virulence factors belonging to ESAT-6 like family of proteins. Furthermore, proteogenomic analysis enabled us to identify 63 novel proteins coding genes and correct 25 existing gene models in H37Ra genome. A majority of these were found to be conserved in the virulent strain H37Rv as well as in other mycobacterial species suggesting that the differences in the virulent and avirulent strains of $M$. tuberculosis are not entirely dependent on the expression of certain proteins or their absence but may possibly be ascertained to functional changes.

Keywords: next-generation sequencing, orbitrap, genome annotation, virulence attenuation, multiomics

\section{INTRODUCTION}

Among the M. tuberculosis strains, H37Ra, derived from M. tuberculosis $\mathrm{H} 37$ clinical isolate has been widely used as a reference strain to study virulence attenuation mechanisms (Zheng et al., 2008). Owing to its avirulent properties, it was used initially to develop tuberculosis vaccines in various animal models (Collins, 2000). Despite multiple efforts, the mechanisms of virulence attenuation are still not completely understood (Philips and Ernst, 2012). Identification of the factors involved in these processes is therefore crucial to understand the mechanisms of immune evasion and host persistence. 
Several genomic differences including insertion-deletions (InDels) and single nucleotide variations (SNVs) have been reported in $\mathrm{H} 37 \mathrm{Ra}$ and its virulent counterpart $\mathrm{H} 37 \mathrm{Rv}$ using comparative genomic analysis (Brosch et al., 1999; Collins, 2000; Zheng et al., 2008). Although some of these variations have been attributed to pathogenicity and virulence (Zheng et al., 2008), the effects of most genomic differences on gene products have not yet been clearly demonstrated. While the reference genome of $\mathrm{H} 37 \mathrm{Rv}$ has undergone several revisions to date, the genome of H37Ra has only recently been resequenced. Based on the reanalysis, almost $50 \%$ of the differences have been accredited to genome sequencing errors thereby suggesting that the genome of H37Ra is significantly more similar to $\mathrm{H} 37 \mathrm{Rv}$ and that variations earlier attributed to virulence are no longer viable (Elghraoui et al., 2017).

At the proteome and transcriptome level, numerous studies have comprehensively analyzed changes in several M. tuberculosis virulent strains (Målen et al., 2011; Jena et al., 2013; Jhingan et al., 2016) including expression profiling of reference and mutant strains, identification of non-coding RNAs and mapping of transcriptional start sites to the genome of $\mathrm{H} 37 \mathrm{Rv}$ and other clinical isolates (Bifani et al., 2000; Karim et al., 2011; Cortes et al., 2013; Periwal et al., 2015). However, studies involving H37Ra have been mostly comparative and focusing primarily on the differences in virulence and pathogenicity determining factors. Comparative proteomic analysis of membrane proteome by Målen et al. (2011), whole cell analysis by Jhingan et al. (2016) and an in silico approach by Jena et al. (2013) have revealed several differentially expressed proteins in H37Ra with respect to H37Rv. In addition to proteins which are distinct to each strain, they also reported proteins in H37Ra carrying mutations that may be associated with virulence attenuation. A comparative proteomic study recently published by our group (Verma et al., 2017) also revealed a distinct pattern of expression of proteins in the two strains of M. tuberculosis. Additionally, distinct phosphorylationmediated changes in type VII bacterial secretion system, twocomponent regulatory system and fatty acid biosynthesis were observed in H37Ra compared to H37Rv. This suggests that there are significant changes in the functional proteome between the two strains despite their origin from the same parental strain. Most of these studies reported the absence of proteomic evidence for several genes in H37Ra with some being attributed to the mechanisms of virulence and pathogenicity. Regardless of the extensive literature on the subject, a detailed analysis of the expression profile of H37Ra is currently unavailable.

Using an integrated OMICs approach, we have earlier successfully demonstrated the utility of proteomic analysis in the refinement of genome annotations of several clinically important organisms (Chaerkady et al., 2011; Kelkar et al., 2011; Renuse et al., 2011; Potgieter et al., 2016; Prasad et al., 2017). In this study, we report a multi-OMICs approach for the indepth characterization of $M$. tuberculosis H37Ra. In addition to

Abbreviations: BLAST, Basic Local Alignment Search Tool; PSM, Peptide Spectrum Match; FDR, False Discovery Rate; ICDS, Interrupted Coding Sequences; GSSP, Genome Search Specific Peptides; iBAQ, intensity Based Absolute Quantification; NCBI, National Centre for Biotechnology Information. confirming the presence of previously described proteins, our study provides both transcriptional and proteomic evidence for genes that were hitherto reported to be absent in H37Ra. We also report the identification of several novel peptides using an in-house developed proteogenomic analysis pipeline that enabled the correction of genome assembly errors and refinement of gene structures.

\section{MATERIALS AND METHODS}

\section{M. tuberculosis Culture}

M. tuberculosis H37Ra strain (MTCC 300) was cultured in Middlebrook 7H9 culture medium (BD) supplemented with OADC (Oleic acid, dextrose and catalase). The tubercle bacilli culture were incubated at $37^{\circ} \mathrm{C}$ in a roller bottle shaker and were harvested.

\section{Genomic DNA Extraction and Whole Genome Sequencing}

Logarithmic phase culture of $M$. tuberculosis H37Ra were harvested by centrifugation at 3,500 rpm for $5 \mathrm{~min}$. The bacterial pellet was lysed in Tris-EDTA-Glucose buffer containing Lysozyme at $37^{\circ} \mathrm{C}$ for $2 \mathrm{~h}$, followed by denaturation of proteins using SDS and proteinase $\mathrm{K}$ at $55^{\circ} \mathrm{C}$ for $40 \mathrm{~min}$. Delipidation was done using cetrimide saline solution at $65^{\circ} \mathrm{C}$ for $10 \mathrm{~min}$. DNA was extracted into the aqueous phase post-chloroform/isoamyl alcohol extraction. DNA was precipitated from the aqueous phase using isopropanol. Ethanol washed genomic DNA was resuspended in TE buffer for further use. $100 \mathrm{ng}$ of genomic DNA was used for the construction of sequence library. The DNA library was prepared according to manufacturer's specifications. $100 \mathrm{bp}$ paired-end sequencing was carried out using Illumina HiSeq2500 platform (Illumina Inc., San Diego, USA) (Sharma et al., 2017).

\section{RNA Extraction and RNA-Seq Analysis}

$0.8 \mathrm{OD}$ cultures were harvested in 4M GITC solution. Bacterial pellets were resuspended in Trizol LS and $0.1 \mathrm{~mm}$ zirconia beads. Cells were lysed by 3 pulses of bead beating at 4,800 rpm thrice for $30 \mathrm{~s}$. The cell lysates were extracted with chloroform: isoamyl alcohol and RNA was precipitated from the aqueous phase. RNA was DNase treated, followed by column purification. Construction of cDNA libraries was carried out following manufacturers' instructions. Amplified cDNA fragments were sequenced using Illumina HiSeq2500 platform (Illumina Inc., San Diego, USA) (Sreenivasamurthy et al., 2017).

\section{Protein Extraction and Sample Preparation for Proteomic Analysis}

For proteomic analysis, the bacterial pellets were lysed using $4 \%$ SDS, $50 \mathrm{mM}$ TEABC, $\mathrm{pH} 8.0$ with heating $95^{\circ} \mathrm{C}$ for $20 \mathrm{~min}$. The samples were then sonicated using a water bath sonicator for $60 \mathrm{~min}$ and centrifuged at 13,000 rpm for $10 \mathrm{~min}$ (Verma et al., 2017). The protein concentration in the lysate was determined using bicinchoninic acid assay (Pierce, Waltham, MA). Two different fractionation methods were employed. In the first method, $200 \mu \mathrm{g}$ of proteins was 
resolved on $10 \%$ SDS-PAGE gel, and 16 gel bands were excised and destained. Proteins in the gel bands were reduced and alkylated using $5 \mathrm{mM}$ dithiothreitol (DTT) at $60^{\circ} \mathrm{C}$ for $20 \mathrm{~min}$ and $20 \mathrm{mM}$ iodoacetamide (IAA) at room temperature for $10 \mathrm{~min}$ in the dark respectively. Trypsin (modified sequencing grade; Promega, Madison, WI, USA) was added to the gel bands, and digestion was carried out at $37^{\circ} \mathrm{C}$ for $16 \mathrm{~h}$. The peptides were extracted and subjected to desalting using $\mathrm{C}_{18}$ StageTips.

In-solution digestion was carried out using one $\mathrm{mg}$ of the protein lysate followed by HPLC-based fractionation. The protein lysate was reduced and alkylated as described earlier. The protein pellet devoid of SDS was obtained by precipitation using six volumes of ice-cold acetone at $-20^{\circ} \mathrm{C}$ for $6 \mathrm{~h}$ followed by centrifugation. The protein pellet was resuspended in $50 \mathrm{mM}$ TEABC, and enzymatic digestion was carried out by adding trypsin (Worthington Biochemical Corporation, Lakewood, NJ, USA) at a final concentration of $1: 20(\mathrm{w} / \mathrm{w})$ for $16 \mathrm{~h}$ at $37{ }^{\circ} \mathrm{C}$. The peptide digest was loaded onto Waters XBridge column (Waters Corporation, Milford, MA; $130 \AA, 5 \mu \mathrm{m}, 250 \times$ $4.6 \mathrm{~mm}$ ) using a manual injector attached to Hitachi HiChrom HPLC system. Fractionation of the peptide digest was carried out using a $130 \mathrm{~min}$ gradient, at a flow rate of $0.5 \mathrm{ml} / \mathrm{min}$ of solvent A (10 mM TEABC buffer, $\mathrm{pH} \sim 8.5)$ and solvent B (10 mM TEABC buffer, 90\% acetonitrile, $\mathrm{pH} \sim 8.5$ ). The gradient program was set as follows: $97 \%$ A for $20 \mathrm{~min}, 90 \%$ A for $60 \mathrm{~min}$, a gradient of $35-100 \%$ of $\mathrm{B}$ for $25 \mathrm{~min}$ and further subjected to equilibration in $95 \%$ of solvent $\mathrm{A}$ for $25 \mathrm{~min}$. The fractionated sample was finally concatenated into 24 fractions. Pooled samples were lyophilized and desalted using $\mathrm{C}_{18}$ StageTips.

\section{LC-MS/MS Analysis}

The peptides obtained from a total of 30 fractions were subjected to LC-MS/MS analysis using Orbitrap Fusion Tribrid mass spectrometer (Thermo Fisher Scientific, Bremen, Germany) coupled to Easy-nLC1200 nano-flow UHPLC (Thermo Scientific, Odense, Denmark). The peptide digests were reconstituted in $0.1 \%$ formic acid and loaded onto nanoViper trap column $2 \mathrm{~cm}$ ( $3 \mu \mathrm{m} \mathrm{C18} \mathrm{Aq)} \mathrm{(Thermo} \mathrm{Fisher} \mathrm{Scientific).} \mathrm{Peptide} \mathrm{separation}$ was carried out using EASY-Spray C18 analytical column $(15 \mathrm{~cm}, 75 \mu \mathrm{m}$ PepMap C18, $2 \mu \mathrm{m}$ C18 Aq) (Thermo Fisher Scientific) at a flow rate of $300 \mathrm{~nL} / \mathrm{min}$. A linear gradient of $5-35 \%$ solvent B ( $80 \%$ acetonitrile in $0.1 \%$ formic acid) over $100 \mathrm{~min}$ was used to resolve the peptide mixture with a total run time of $120 \mathrm{~min}$ including sample loading and column reconditioning. Data dependent acquisition with full scans in $400-1,600 \mathrm{~m} / \mathrm{z}$ range was carried out using an Orbitrap mass analyzer at a mass resolution of 120,000 at $200 \mathrm{~m} / \mathrm{z}$. The most intense precursor ions from a survey scan were selected for MS/MS and fragmented using HCD mode with $34 \%$ normalized collision energy and detected at a mass resolution of 30,000 at $200 \mathrm{~m} / \mathrm{z}$. Peptide charge state was set to 2-6, and dynamic exclusion was set to $30 \mathrm{~s}$. Internal calibration was carried out using lock mass option (m/z 445.1200025) from ambient air.

\section{DATA ANALYSIS}

\section{Mapping and Variant Detection Using M. tuberculosis H37Ra and H37Rv Reference Genomes}

The quality of the raw reads obtained from whole genome sequencing analysis was verified using FastQC. The filtered reads were aligned to $\mathrm{H} 37 \mathrm{Ra}$ reference genome (NC_009525.1) (downloaded from NCBI, updated May, 2016) (Zheng et al., 2008) and NZ_CP016972.1 (downloaded from NCBI, updated March, 2017) (Elghraoui et al., 2017) using Burrows-Wheeler Alignment Tool (BWA version-0.7.15) (Li and Durbin, 2009). The alignment files were subjected to local realignment and de-duplication using the Genome Analysis Toolkit (GATK version-3.6). SNVs in the coding and promoter region were identified from each alignment file using GATK (McKenna et al., 2010). Insertion/Deletions (In-Dels) were identified from each alignment file using GATK and Pindel (version-0.2.5b8) (Ye et al., 2009). The variants and In-Dels were annotated using in-house Perl scripts. Briefly, the SNVs were annotated using reference genome fasta file and gtf file to identify if they were falling in the region of protein-coding genes. Further, these variants were categorized as synonymous and nonsynonymous SNVs with amino acid change and its corresponding position were also obtained. The In-Dels were classified into insertions and deletions with the details of protein coding genes and the number of bases inserted or deleted. All variants identified in this study were manually inspected using Integrative Genomics Viewer (IGV version- 2.3.86) (Robinson et al., 2011). The data was also compared with $\mathrm{H} 37 \mathrm{Rv}$ reference genome to identify H37Ra specific variants with respect to $\mathrm{H} 37 \mathrm{Rv}$. The raw reads after quality check was aligned to $M$. tuberculosis $\mathrm{H} 37 \mathrm{Rv}$ reference genome (NC_000962.3) using the methods described above. The SNVs and In-Dels were then compared with genetic variations reported by Zheng et al and Elghraoui et al. to identify similarities and differences in identifications.

\section{Transcriptome Data Analysis}

Reads obtained from the RNA-Seq analysis were quality filtered for Phred-based base quality $(\mathrm{Q}>20)$ using FastQC. The reads that passed the quality threshold were used in downstream analysis steps. BWA (version 0.7.15) with default parameters was used to align the reads against the M. tuberculosis H37Ra reference genome (NC_009525.1) (updated May, 2016). For the coding transcriptome, the number of reads mapped to each coding sequence (CDS) was calculated using HTseq (Anders et al., 2015) and corrected for gene length and library depth to generate normalized reads per kilobase per million mapped reads (RPKM) values (Mortazavi et al., 2008).

\section{Database Searches for Peptide and Protein Identification}

Raw data files obtained from mass spectrometry analysis were processed using Proteome Discoverer software version 2.1 (Thermo Fisher Scientific, Bremen, Germany). The data were searched using SequestHT and Mascot search algorithms against H37Ra protein database (downloaded from NCBI, updated May, 
2016) combined with common contaminants (total number of sequences: 4,143 proteins). The search parameters employed for the analysis included trypsin as the protease with a maximum of 2 missed cleavages allowed. Oxidation of methionine and protein $\mathrm{N}$-terminal acetylation were set as dynamic modifications, whereas, carbamidomethylation of cysteine was specified as static modification. Precursor ion mass tolerance and fragment ion mass tolerance were set to $10 \mathrm{ppm}$ and $0.05 \mathrm{Da}$ respectively. Percolator node was employed to calculate False Discovery Rate (FDR). The FDR for the proteomic data in the study was $<1 \%$ at both peptide and PSM levels. The global protein FDR for the entire dataset is $4 \%$.

\section{Generation of Custom Databases for Proteogenomics Analysis}

For the proteogenomic analysis, four custom databases were generated including (i) six-frame translated genome database, (ii) three-frame translated RNA-Seq transcript database, (iii) alternative start codon database, and (iv) a hypothetical $\mathrm{N}$ terminal peptide database. The six-frame translated genome database was created using the genome sequence of $\mathrm{H} 37 \mathrm{Ra}$ (NCBI Reference Sequence: NC_009525.1) downloaded from the NCBI FTP site. Using in-house python scripts, six-frame translated database was created containing genomic sequences translated from stop codon to stop codon using standard (confirm) codon table. The information pertaining to the start coordinate, end coordinate and the frame of translation were listed in the header of the translated amino acid sequence. Only those translated sequences were considered which had peptide sequences with the length of $\geq 7$ amino acids. The translated transcriptome database was created using assembled transcripts from RNAseq data generated in this study. Mycobacterium species are known to use GTG and TTG as alternate initiator methionine codons. Therefore, to create an alternative start codon database, the genome was translated in six frames, and wherever GTG and TTG codons were encountered, they were considered as probable translation start sites and translated as the initiator methionine in addition to valine and leucine (Kelkar et al., 2011). The hypothetical N-terminal tryptic peptide database was created by fetching all the peptide sequences that began with methionine and ended with $K / R$ from the six-frame translated genome database. Common contaminant sequences were added to each database, and only those peptide sequences with lengths ranging from 7 to 25 amino acids were included in the databases. The search parameters employed were the same as discussed in the previous section.

\section{Proteogenomic Analysis and Workflow for Manual Genome Annotation}

The unassigned spectra obtained after searches against the reference protein database were used for proteogenomic analysis. The data were searched against the custom databases mentioned in the previous section. Peptides obtained after applying the $1 \%$ FDR cutoff criteria were selected, and genomic coordinates were obtained for these using an in-house Perl script. Briefly, the peptide sequences were mapped to the six frame translated genome fasta file and the genomic coordinates of the translated sequence was retrieved. Based on the start coordinate, end coordinate and the frame of translation, genomic coordinates for the peptides were obtained. Peptides mapping to multiple regions in the $\mathrm{H} 37 \mathrm{Ra}$ genome and those mapping to known protein sequences were not considered for further analysis. Genome search specific peptides were categorized as follows: (1) mapping to intergenic region, (2) partially overlapping with annotated genes, and (3) completely mapping to annotated genes but translated in a different frame. Alternative gene models were predicted using two gene prediction programs-FgeneSB and GeneMark (Version 2.5) (Besemer and Borodovsky, 2005). Orthologous evidence for putative novel genes and refined gene models were obtained by sequence comparison across Mycobacterium species using protein BLAST (https://blast.ncbi. nlm.nih.gov/Blast.cgi). MS/MS spectra of all instances of peptides suggesting novel gene models or refinement of existing gene models were manually verified.

\section{Estimation of Protein Abundance and Gene Ontology Analysis}

The relative abundances of identified proteins in H37Ra were determined using intensity-based absolute quantitation method (iBAQ) (Schwanhäusser et al., 2011). The intensities of all the observed tryptic peptides of a given protein were summed and then divided by the number of theoretically observable tryptic peptides. Gene Ontology-based functional classification information for molecular function, biological process, and subcellular localization were fetched from Uniprot for all the proteins present in the protein database. FunRich tool was used for functional enrichment of the identified proteins in our study using the Uniprot annotation as backend database (Pathan et al., 2015).

\section{Data Availability in Public Repositories}

The sequencing data from whole genome sequencing and transcriptomic profiling experiment have been deposited in the SRA database (www.ncbi.nlm.nih.gov/sra). They can be accessed using identifiers SRP127305. The mass spectrometry data have been deposited to the ProteomeXchange Consortium (http://proteomecentral.proteomexchange.org) via the PRIDE partner repository with the dataset identifier PXD0 08555 .

\section{RESULTS}

In the current study, a multi-pronged OMICs approach was employed for the in-depth characterization of $M$. tuberculosis H37Ra (Figure 1A). The details of the analysis carried out in this study is summarized in Figure 1B.

\section{Genomic Features of H37Ra}

To identify the genomic features of $M$. tuberculosis H37Ra strain (MTCC300), we carried out whole genome sequencing analysis on Illumina HiSeq2500 platform. The genome of M. tuberculosis H37Ra comprises of 4,419,977 bp encoding a total of 4,152 protein-coding sequences (CDS). On an average, 
A

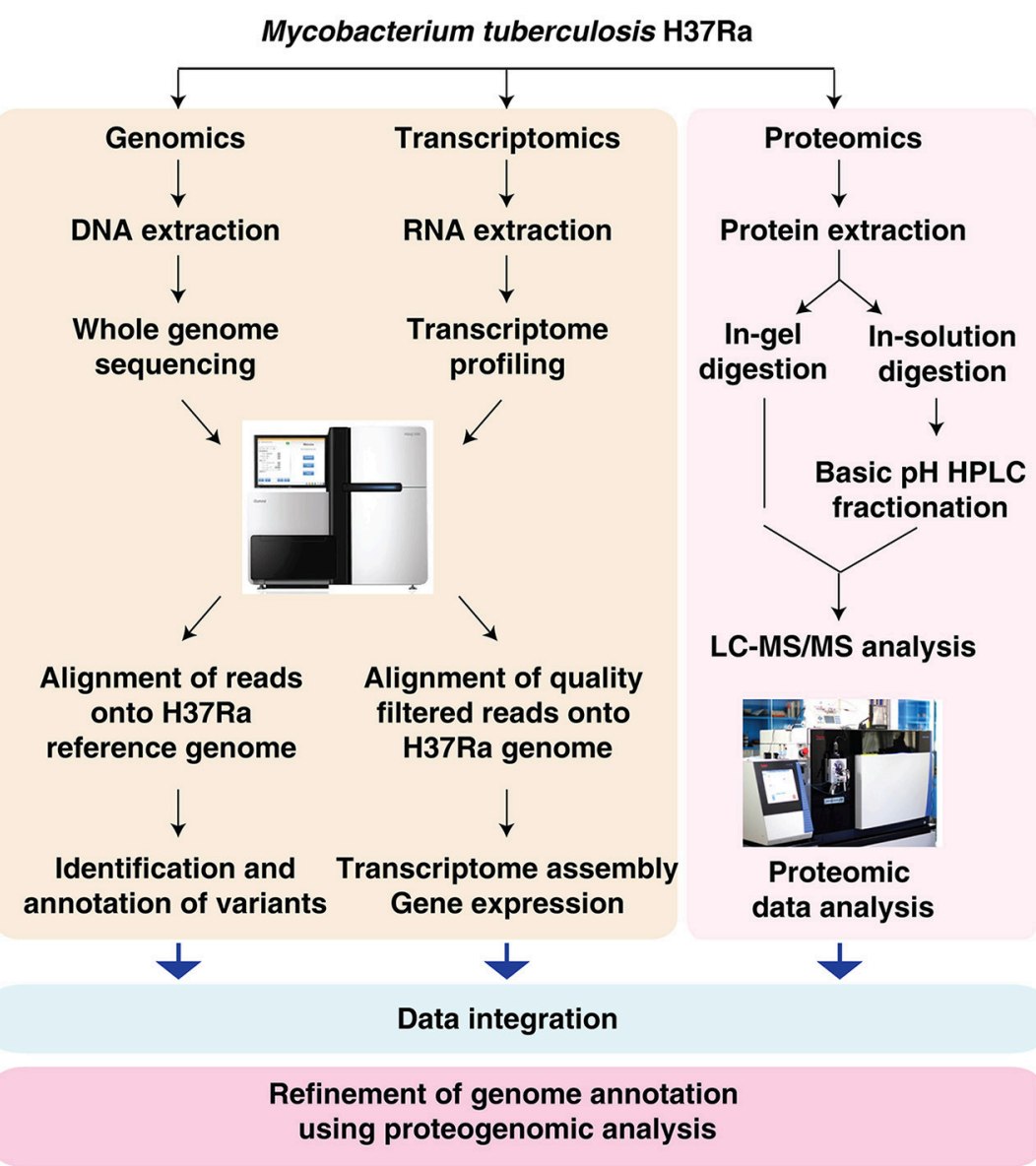

B

\begin{tabular}{cccc}
\hline & Data acquisition platform & Data output & Summary \\
\hline Genome & Illumina HiSeq2500 & 4.4 million reads & $\begin{array}{c}4.2 \text { million } \\
\text { aligned reads }\end{array}$ \\
RNA-Seq & Illumina HiSeq2500 & 87 million reads & $\begin{array}{c}68 \text { million } \\
\text { aligned reads }\end{array}$ \\
Proteome & $\begin{array}{c}\text { Thermo Orbitrap Fusion } \\
\text { Tribrid mass spectrometer }\end{array}$ & 0.7 million spectra & $\begin{array}{c}355,000 \text { peptide } \\
\text { spectrum matches }\end{array}$
\end{tabular}

FIGURE 1 | Characterization of Mycobacterium tuberculosis H37Ra using an integrated OMICS analysis. (A) Schematic representation of the workflow employed in the current study to characterize the genome repertoire of Mycobacterium tuberculosis H37Ra. (B) Summary of genomic, transcriptomic and proteomics analysis of Mycobacterium tuberculosis H37Ra.

we obtained 4.4 million sequence reads at a read depth of $100 \mathrm{X}$ with coverage of $\geq 95 \%$. The raw reads obtained from the analysis were aligned to H37Ra reference genome (NC_009525.1) and H37Ra genome (NZ_CP016972.1) analyzed by Elghraoui and group (Elghraoui et al., 2017). In all, we identified 58 SNVs, 5 insertions and 12 deletions with respect to NC_009525.1 (Supplementary Tables 1A,B) and 13 SNVs, 2 insertions and 3 deletions with respect to NZ_CP016972.1 (Supplementary Tables 1C,D).
We further compared our data with $\mathrm{H} 37 \mathrm{Rv}$ reference genome (NC_000962.3) which resulted in the identification of 66 SNVs (Supplementary Table 2A), 16 insertions and 11 deletions (Supplementary Table 2B). We also identified mutations in the promoter regions of 7 genes (Supplementary Table 2C) including OtsB (Rv2006), a key enzyme in the trehalose biosynthesis pathway that is required for the synthesis of trehalose-based glycolipids including diacyltrehalose/polyacyltrehalose (DAT/PAT) and sulfolipid (SL). 
These mycolipids are essential constituents of mycobacterial cell wall and therefore play an important role in virulence (Kalscheuer and Koliwer-Brandl, 2014, Murphy et al., 2005). It is important to note that H37Ra fails to produce acyltrehalose which is an abundant lipid in H37Rv (Chesne-Seck et al., 2008). This lack of acyltrehalose was previously shown to be due to loss of function mutation in the PhoR transcription factor preventing transcription of the $p k s 2-3 / 4$ locus. Surprisingly mutations in pks2-3/4 do not lead to loss of virulence in H37Ra. Our data therefore suggests that mutations that affect other metabolites in the pathway may contribute to loss of virulence rather than a direct effect on acyltrehalose.

Of the total number of mutations identified in $\mathrm{H} 37 \mathrm{Ra}$ with respect to $\mathrm{H} 37 \mathrm{Rv}$ genome, $27 \mathrm{SNVs}$ and two of each insertions and deletions were identified for the first time (Supplementary Tables 2A,B).

In order to identify high confidence H37Ra-specific variants, we next compared SNVs and In-Dels common to all three studies. Our analysis led to the confirmation of 39 single nucleotide polymorphisms common across all the three studies including variations in phoP (S219L) and espK (I228M) (Figure 2, Supplementary Tables 3A,B). We also confirmed the stop gain mutations in phosphate $\mathrm{ABC}$ transporter permease -PstA and molybdopterin oxidoreductase. Of the earlier reported In-Dels, 14 insertions, and 9 deletions were common to all three studies validating the fact that these mutations are indeed actual changes in the avirulent $\mathrm{H} 37 \mathrm{Ra}$ strain (Figure 2, Supplementary Table 3B). These include large deletions in $n r d H$-redoxin, IS6110 transposase and IS3 family transposase.

\section{Transcriptomic Profile of H37Ra}

Transcriptome profiling of M. tuberculosis H37Ra was carried out on Illumina-HiSeq-2500 sequencing platform using pairedend sequencing (100 bp paired-end reads). From the RNA-Seq analysis, 87,862,710 reads were identified resulting in $78 \%$ read alignment to the reference genome (NC_009525.1). The number of reads mapping to each coding sequence (CDS) was calculated and corrected for gene length and library depth to generate normalized reads per kilobase per million mapped reads (RPKM) values (Mortazavi et al., 2008). A total of 3,945 CDS had RPKM values of 1 or more representing $94 \%$ of the annotated genome (Figure 2, Supplementary Table 4). The RPKM values for the genes in which we identified promoter mutations were varied. The transcript level expression of OtsB falls in the lower 10\%, whereas that of $M f t E$ falls in the higher $10 \%$ of the overall expression range. Additionally, transcriptomic evidence was also obtained for genes that were missed in the earlier annotation (described in Proteogenomics section).

\section{Proteomic Landscape of $M$. tuberculosis H37Ra}

In order to achieve maximum proteome coverage, we employed two fractionation techniques namely in-gel digestion and high pH HPLC-based -fractionation. The mass spectrometry-derived data were analyzed using SequestHT and Mascot search engines available through the Proteome Discoverer software suite (version 2.1). A total of $743,816 \mathrm{MS} / \mathrm{MS}$ spectra were acquired in FT-FT mode resulting in the identification of 344,436 peptide spectral matches mapping to 31,122 peptides. Overall, these peptides corresponded to 3,205 proteins, the largest report on $\mathrm{H} 37 \mathrm{Ra}$ to date. Altogether, the identified proteins correspond to $\sim 77.4 \%$ of H37Ra reference proteome (Figure 2). The complete lists of peptides and proteins identified in this study are provided in Supplementary Table 5A.

To determine the extent of correlation between mRNA abundance and protein expression, we compared the proteome data of H37Ra with its transcriptome. Of the total transcripts mapped to $\mathrm{H} 37 \mathrm{Ra}$ proteome, $80 \%$ of the transcriptome was found to have corresponding peptide evidence (Figure 3A). In addition, we identified 20 proteins exclusively through proteomic analysis and 763 transcripts exclusive from the transcriptomics data. To obtain an estimate of the protein abundance, we employed iBAQ algorithm. Spearman's correlation coefficient was computed to correlate protein and mRNA data using log-transformed iBAQ values and FPKM-based transcript abundances. A Spearman's correlation coefficient of $(r)=0.47)$ (Figure 3B) was observed corroborating with previous reports that the proteome expression does not necessarily correlate with the transcript level of expression.

\section{Functional Annotation of the H37Ra Proteome}

The identified proteins were categorized based on their molecular function, cellular localization and role in biological processes using the FunRich tool (Pathan et al., 2015; Figure 3C). Biological process-based classification revealed the enrichment of proteins involved in transcription (10.5\%) and its regulation (9.7\%). Further, molecular function-based classification showed ATP binding $(13.7 \%)$ to be the most augmented function. Proteins such as NusA, a transcription termination/antitermination protein, TetR-family transcriptional regulator and LuxR family transcriptional regulator were among the ones found enriched in biological process-based classification. Molecular chaperones including GroES, GroEL and ATP synthase subunits were also found to be significantly enriched. Cellular componentbased classification indicated that most proteins were localized to the cytoplasm (25.8\%) followed by membrane. Examples of proteins localized to the cytoplasm included chromosomal replication initiator protein DnaA, Cyclopropane mycolic acid synthase 1 which play important roles in DNA replication initiation and determining cell envelope permeability, host immunomodulation, and persistence, respectively. We next looked at the abundance distribution of the proteome based on the iBAQ method (Figure 4A). The top 10\% of abundant proteins were dominated by proteins involved in protein synthesis, protein folding, and energy metabolism processes. However, bottom $10 \%$ of least abundant proteins were found to be specifically enriched for proteins localized in the membrane with transporter activity. We also found enrichment of enzymes involved in cobalamin synthesis and proteins with monooxygenase activity. 


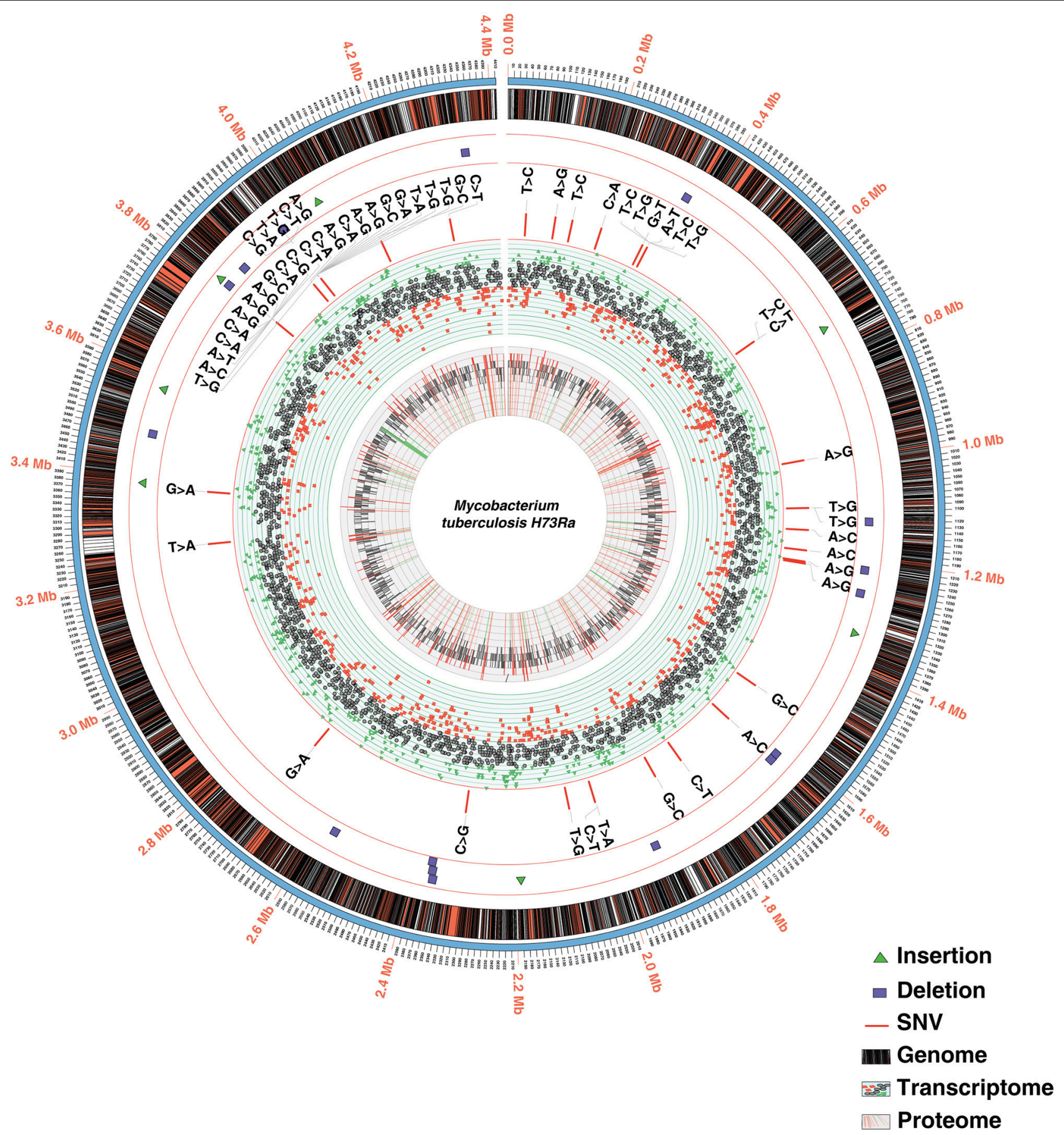

FIGURE 2 | Circos plot depicting the integrated view of the data obtained through multi-OMICs approach.

\section{Evidence for Protein-Coding Genes Hitherto Thought to be Absent in H37Ra}

In an earlier study reported by He et al. (2003), two-dimensional gel electrophoresis-based comparative proteome analysis of culture supernatant proteins obtained from H37Ra and $\mathrm{H} 37 \mathrm{Rv}$ was performed to examine differential expression of proteins among the two strains. The analysis resulted in the identification of 5 proteins to be exclusively identified in $\mathrm{H} 37 \mathrm{Rv}$ in comparison to H37Ra. These include members of the ESX protein family namely Rv2346c (esxO), Rv2347c (esxP), Rv1038c (esxJ), Rv1197 (esxK), and Rv3620c (esxW). On the basis of these findings, the authors suggested that these proteins may play an important 
A

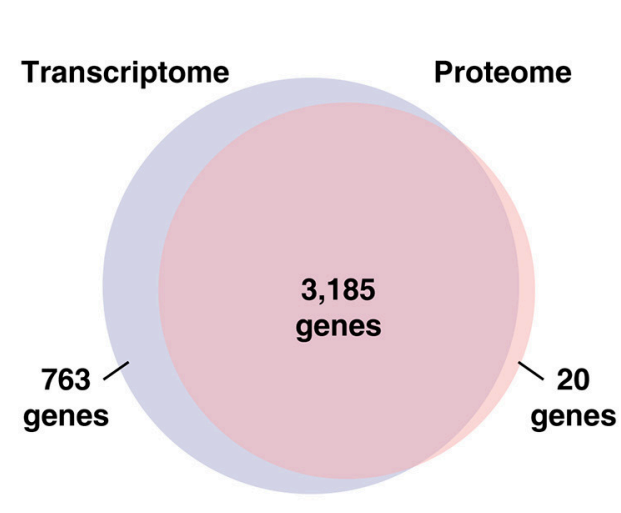

B

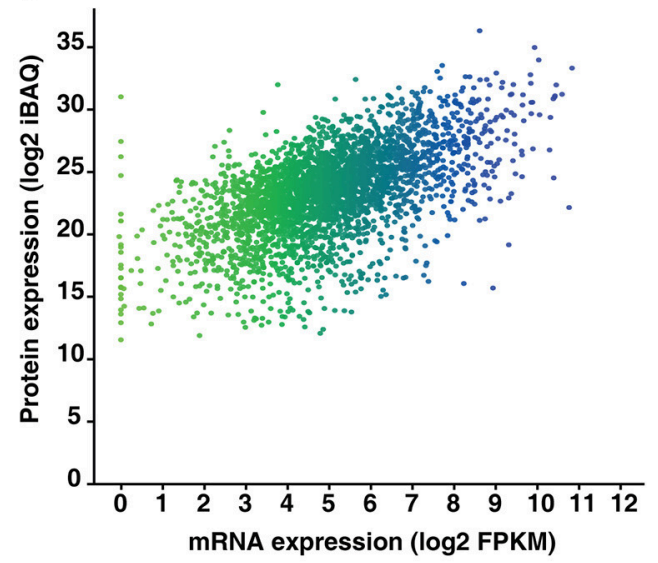

C

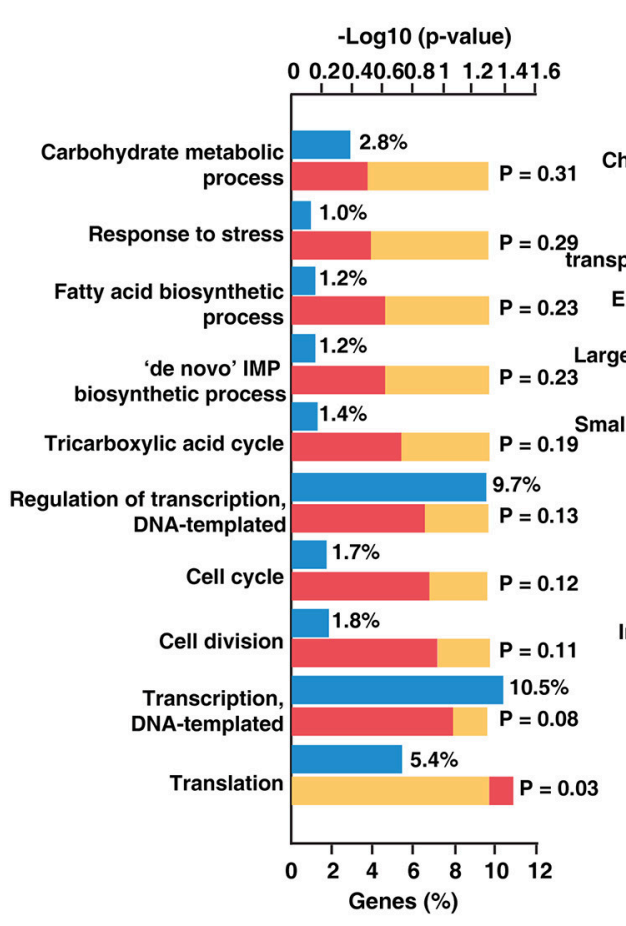

Cellular Component

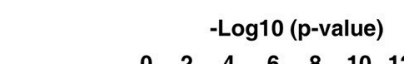

Chromosome

$\begin{array}{lllllll}0 & 2 & 4 & 6 & 8 & 10 & 12\end{array}$

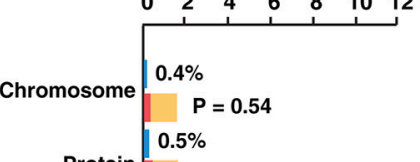

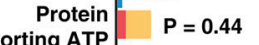

$1.2 \%$

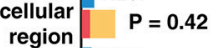

$0.9 \%$

subunit $P=0.23$

all ribosomal $0.9 \%$

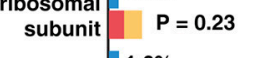

\begin{tabular}{c|c}
$1.0 \%$ \\
Membrane $P=0.19$
\end{tabular}

$2.8 \%$

Cell $P=0.05$

\begin{tabular}{l|l} 
Intracellular & $5.7 \%$ \\
\cline { 2 - 2 } & $\mathrm{P}=\mathbf{0 . 0 4}$
\end{tabular}

Ribosome $5.3 \%$

$P<0.01$

Cytoplasm

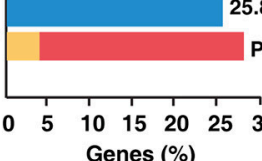

Molecular Function

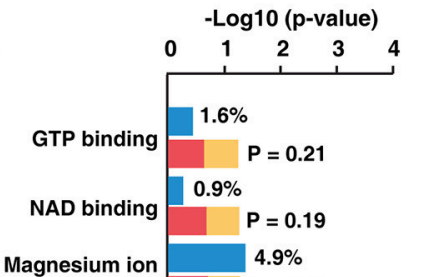

binding $\quad \mathrm{P}=0.19$

FAD binding $\quad 3.8 \%$

\begin{tabular}{ll} 
& $2.0 \%$ \\
\hline
\end{tabular}

$P=0.12$

$3.5 \%$

Zinc ion binding $\quad \mathbf{P}=0.11$

$1.5 \%$

RNA binding $\quad P=0.07$

\begin{tabular}{l|l} 
Structural constituent & $3.3 \%$
\end{tabular}

of ribosome $\quad P=\mathbf{0 . 0 6}$

Pyridoxal phosphate $1.8 \%$

binding $\quad P=0.04$

$<0.001$

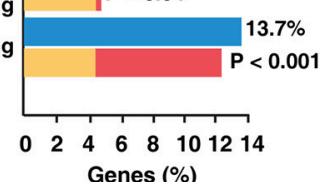

FIGURE 3 | Summary of transcriptomic and proteomic analysis of Mycobacterium tuberculosis H37Ra.(A) Comparison of the mRNA and protein levels reveals $80 \%$ of the genes have both transcriptome and proteome evidence. 763 genes were identified based on transcript evidence alone. (B) Correlation plot depicting concordance between RNAseq and proteomic data for Mycobacterium tuberculosis H37Ra strain. (C) Gene Ontology-based classification of identified proteins based on biological process, molecular function and cellular component.

role in virulence attenuation of H37Ra. From our analysis, we identified transcript evidence for es $x O$, es $x J$, es $x P$, and es $x K$ in the current analysis. We also observed protein expression of esxO and esxK with 5 and 11 peptides respectively (Table 1). Based on the abundance scale, these proteins range in the top $10 \%$ abundance with esxO being expressed the most. Representative
MS/MS spectra are illustrated in Supplementary Figure 1A. The data obtained from this analysis refutes the findings from the previous study with evidence provided at both transcript and proteome level.

We also compared our data with previously published proteomic datasets including Jhingan et al., and Malen et al. 


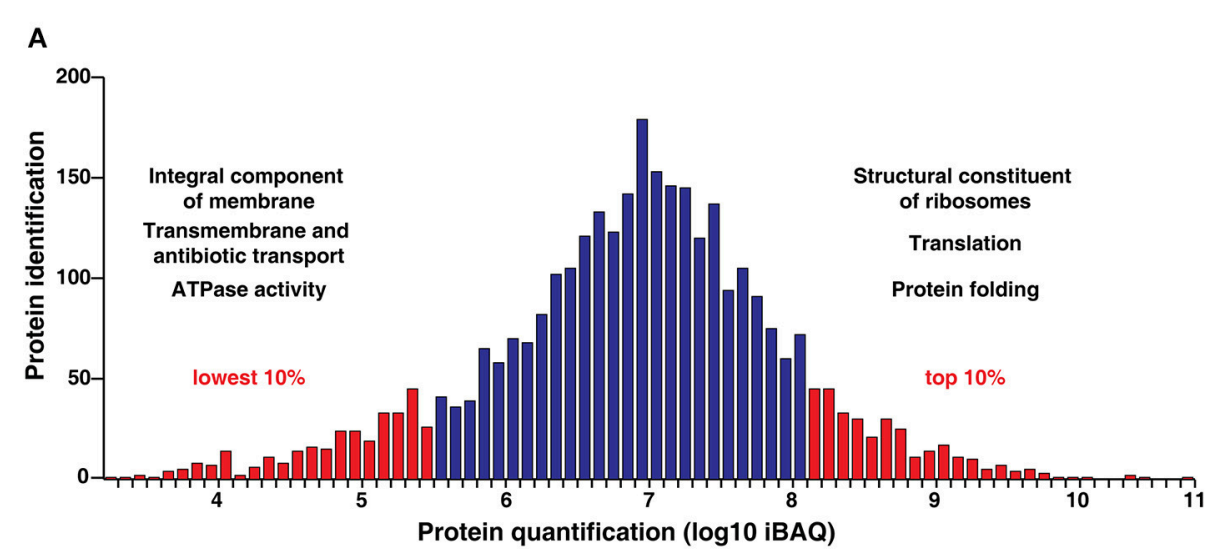

B

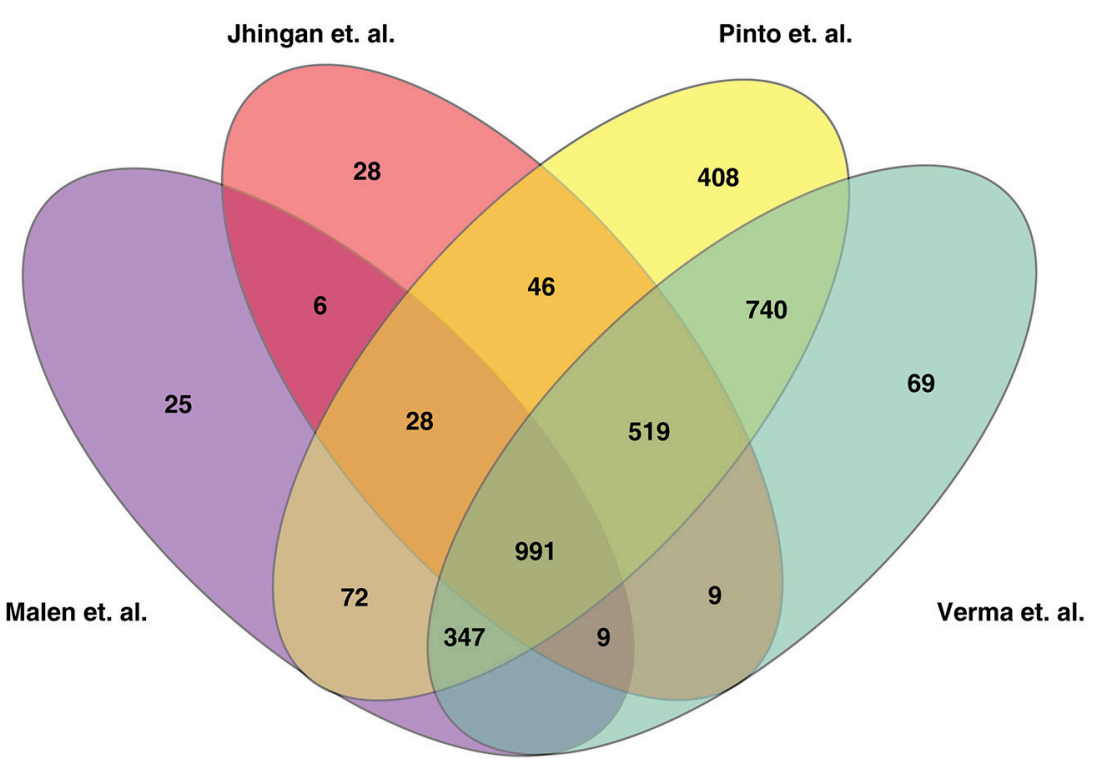

FIGURE 4 | (A) Abundance distribution of the proteins based on the iBAQ method. (The most highly expressed 10\% of proteins are dominated by proteins relating to protein synthesis, protein folding and energy metabolism. The least abundant $10 \%$ of proteins are enriched in proteins with transporter activity). (B) Comparison of proteins identified by Malen et al., Jhingan et al., Verma et al. and our study. We identified 408 proteins which have not been identified in previous datasets.

We identified 1,019 proteins common to all three data sets and 1,148 proteins unique to our data set. Further, comparison of the current proteomic data with a recent study published by our group revealed 408 proteins identified uniquely in this study (Figure 4B, Supplementary Table 5B). In the study by Malen et al., which focused on the comparative analysis of membrane-associated proteins in $M$. tuberculosis $\mathrm{H} 37 \mathrm{Rv}$ and H37Ra strains, 4 proteins were identified with 4 or more peptides in $\mathrm{H} 37 \mathrm{Rv}$ but reported as absent in H37Ra. These include Rv3479, Rv3792, Rv2319c, and Rv1184c. Verma et al., have shown lower expression of Rv3479 and Rv2319c in H37Ra when compared to H37Rv (Verma et al., 2017). We identified Rv1184c which encodes for PE-PPE domain-containing protein only at the transcript level and the orthologous counterparts of 3 proteins with evidence at both transcript and proteome level. These include Rv3479 (MRA_RS18460) which encodes for a membrane protein (Supplementary Figure 1B), arabinofuranosyltransferase (AftA) (Rv3792) involved in the biosynthesis of the mycobacterial cell wall glycan, arabinan and Rv2319c (MRA_RS12320) belonging to Universal stress protein family protein (Supplementary Figure 1C). The expression levels of the former two proteins were in the top $50 \%$ abundances whereas MRA_RS12320 was among the 10\% of least abundant proteins identified (Table 1). The representative MS/MS spectra are depicted in Supplementary Figures 1A-C. In the current version of NCBI, the protein record of MRA_RS18460 (WP_003901652.1) which is the orthologous counterpart of Rv3479 is suppressed as it is no longer annotated on any genome. 
TABLE 1 | List of proteins hitherto reported as absent in H37Ra.

\begin{tabular}{|c|c|c|c|c|c|c|}
\hline MRA_ID (Rv ID) & Gene symbol & Description & Log2 iBAQ & Log2 RPKM & $\begin{array}{c}\text { H37Ra } \\
\text { Log/H37Rv Log }\end{array}$ & $\begin{array}{c}\text { H37Ra stationary/H37Rv } \\
\text { stationary }\end{array}$ \\
\hline MRA_RS12470 (Rv2346c) & esxO & ESAT-6 like protein EsxO & 31.07 & 10.43 & 1.3 & 0.9 \\
\hline MRA_RS12475 (Rv2347c) & esxP & ESAT-6 like protein EsxP & - & 10.39 & - & - \\
\hline MRA_RS05510 (Rv1038c) & esxJ & ESAT-6 like protein EsxJ & - & 5.87 & 2.1 & 1.7 \\
\hline MRA_RS20115 (Rv3792) & aftA & Arabinofuranosyltransferase & 22.30 & 3.98 & 1.2 & 1.0 \\
\hline MRA_RS12320 (Rv2319c) & Rv2319c & Universal stress protein & 18.67 & 2.66 & 0.5 & 0.8 \\
\hline MRA_RS06305 (Rv1184c) & Rv1184c & Exported protein & - & 2.25 & 0.7 & 1.0 \\
\hline
\end{tabular}

We also identified a protein encoded by MRA_RS02220 (WP_003898444.1) exclusively in our dataset with $\log 2 \mathrm{iBAQ}$ value of 27.55 and $\log 2$ RPKM of 6.8 . It is among the $10 \%$ most highly expressed proteins with 3 unique peptides (Supplementary Figure 1D). However, there is no entry at the protein level in the NCBI database owing to the fact that it is no longer annotated in any genome. A BLAST analysis suggested its conservation across various mycobacterial species. Our analysis thereby emphasizes the power of in-depth proteome analysis in its ability to identify coding evidence for computationally predicted genes.

\section{Identification of Putative Virulence Factors}

We next compared our data with the virulence-associated factors listed in Forrellad et al. (2013). Interestingly, a majority of proteins deemed as virulent factors were involved in mycolic acid and lipid synthesis. It has earlier been established that cell wall of M. tuberculosis plays a vital role in its virulence. It displays a wide array of complex lipids and lipoglycans on its surface, which aid the pathogen in host interaction and disease progression (Smith, 2003; Vander Beken et al., 2011). Therefore, the genes responsible for biosynthesis and transport of potential virulence factors are important from an investigative point of view and may offer new drug targets. Changes in these genes at genome, transcriptome or proteome may affect the virulence of $M$. tuberculosis. In order to characterize such changes, we mapped the mutations identified in genome analysis with the virulence-associated protein. Of the 153 proteins, genes corresponding to 6 proteins- carried mutations in $\mathrm{H} 37 \mathrm{Ra}$ with respect to $\mathrm{H} 37 \mathrm{Rv}$ suggesting the role of these genes in $M$. tuberculosis virulence. These include $p s t A 1$, phoP, plcA, plcB, PE_PGRS51 and apoptosis inhibitor protein.

We further compared the list of putative virulence factors with the proteins identified in this study resulting in the identification of 139 proteins. A partial list of proteins is provided in Table 2 . These proteins were further compared with the list of proteins identified in previously published studies. Altogether, our analysis provides proteomic evidence for 12 putative virulence factors identified for the first time in H37Ra proteome. These include RNA polymerase sigma factors sigE and sigL that are also members of the ECF subfamily, FAD-containing monoxygenase mymA, transporter proteins including MmpL8, lipid carrier protein (MRA_RS18785) and ABC transporter permease (MRA_RS18565) among others.

\section{Identification of Novel Protein Coding Genes in H37Ra Genome Using Genome Search Specific Peptides (GSSPs)}

The data obtained from the proteomic study was further analyzed using proteogenomics pipeline to enable refinement of genome annotation. We considered RefSeq dataset as the reference proteome for gene annotation. As described in the methods section, GSSPs identified from the proteogenomics analysis were categorized. In all, we identified 82 novel peptides corresponding to 63 novel protein-coding genes (Supplementary Table 6A). Of these novel ORFs, 10 were supported by two or more peptides whereas the remaining 53 ORFs were supported by only single peptide with acceptable spectra. Those peptides which matched to a non-coding region or were reported to be translated in a different frame were deemed as novel ORFs. Conservation of these predicted genes across mycobacterial and related species was checked using protein BLAST algorithm.

One of the interesting findings was an insertion of a base pair at position 1,341,580 in the M. tuberculosis H37Ra genome resulting in the frameshift in the CDS of MRA_RS06365. The finding corroborates with the insertion reported by Elghraoui et al., as well. The current NCBI annotation has this gene categorized as a pseudogene. However, based on our analysis, we now provide evidence at both transcript and protein level with 7 peptides mapping uniquely to this putative pseudogene. Orthologous evidence suggests that the protein encoded by this gene is a member of the PPE family (PPE 18) and is conserved across mycobacterial species (Figure 5).

\section{Identification of N-Terminal Peptides and Correction of Translational Start Sites (TSS)}

Using alternate codon database and hypothetical N-terminal tryptic peptide database, we identified events of N-terminal extension in 25 proteins (Supplementary Table 6B and 6C). Several proteins which were found to have $\mathrm{N}$-terminal extension were involved in cell wall synthesis and membrane transport. Additionally, using protein N-terminal acetylation as dynamic modification, we validated protein translation start sites of 332 
TABLE 2 | Partial list of putative virulence factors identified in H37Ra.

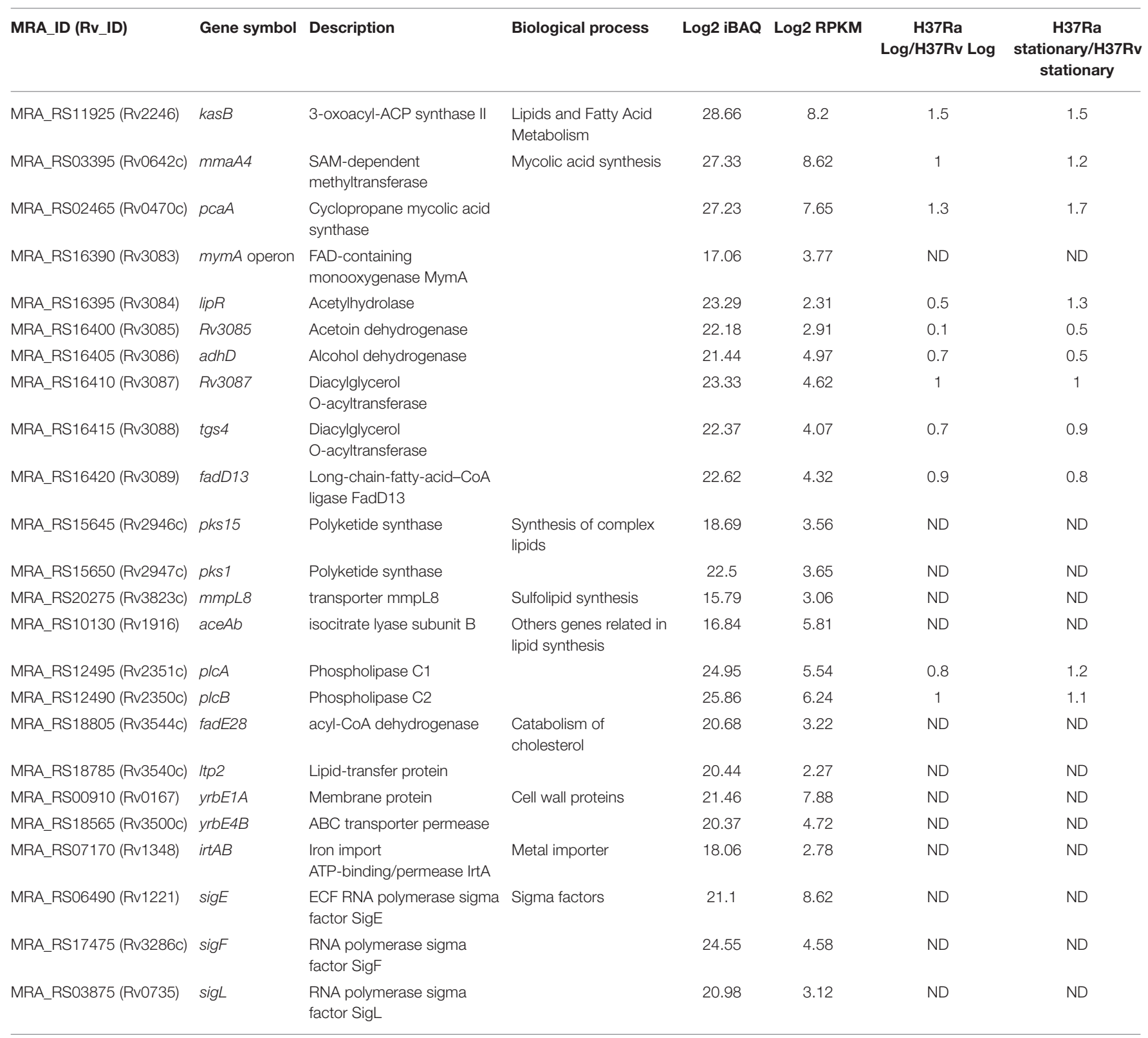

proteins reported in H37Ra reference database (May 2016). These include high abundant proteins from ESX secretion system of mycobacteria such as ESX-1 secretion-associated protein EspB, EspF, and EspJ. We also identified 3 incidences of ICDSs in H37Ra genome of which, one was attributed to a potential sequencing error and the other two to stitching errors.

\section{DISCUSSION}

Integrated analyses of multi-omics data have proven to be powerful tools to understand complex biological questions (Prasad et al., 2017). Whole genome sequencing of M. tuberculosis H37Ra carried out in this study confirmed $39 \mathrm{SNV}$ s that were reported in earlier studies. Interestingly, we identified $46 \%(18 / 39)$ of these mutations in the genes encoding PE-PGRS family proteins (Supplementary Table 3A). The members of the PE-PGRS family have been shown to play a role in evasion of host immune responses, possibly via antigenic variations (Sampson, 2011). Further, we also confirmed stop gain mutations in two H37Ra genes namely phosphate $\mathrm{ABC}$ transporter permease $p s t A$ and oxidoreductase. $p s t A$ is involved in active transport of inorganic phosphate across the membrane (Braibant et al., 2000), oxidoreductase, on the other hand, is involved in cellular respiration (Sassetti et al., 2003). Of the 14 high confidence H37Ra-specific variants previously reported by 


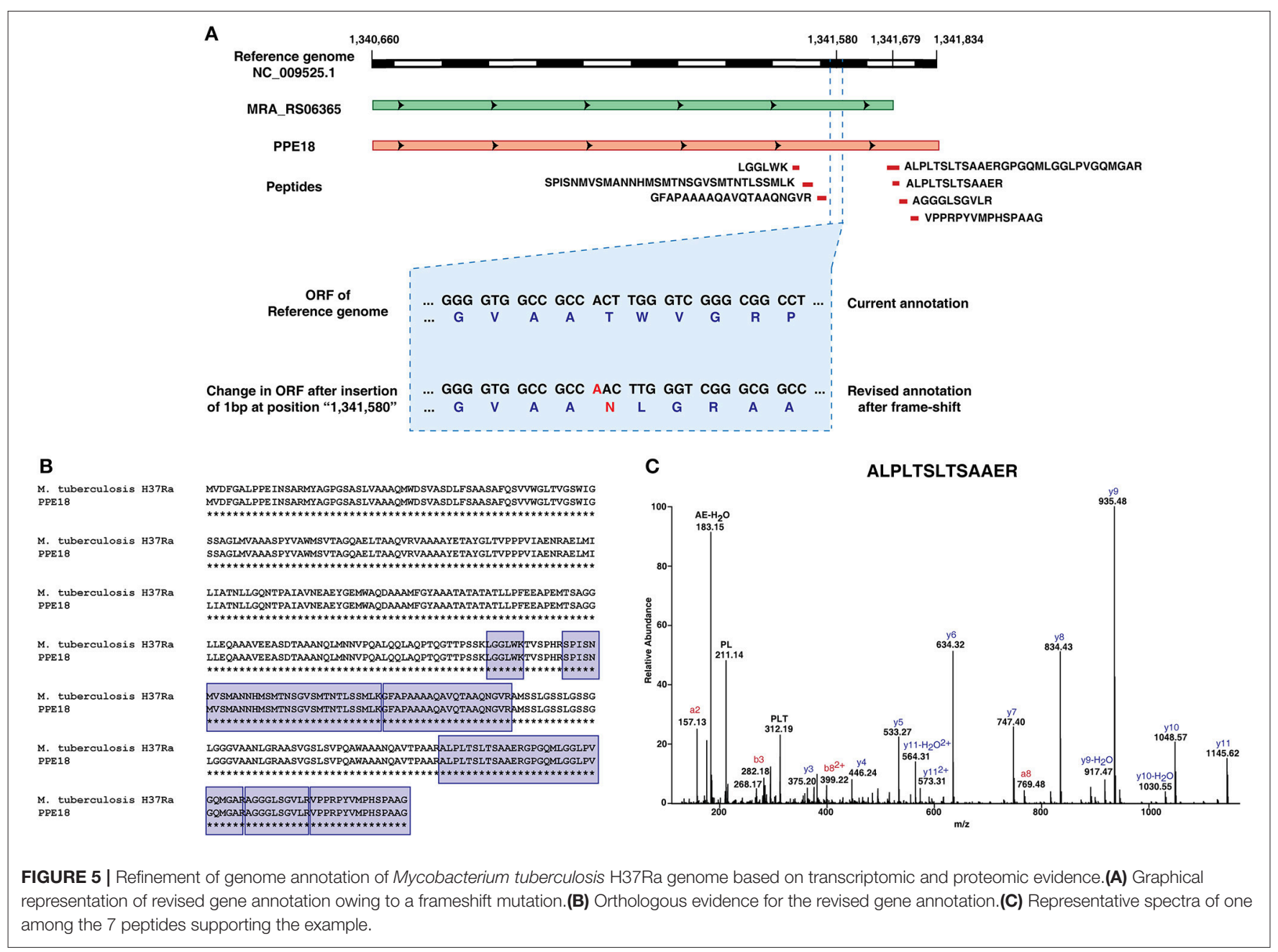

Elghraoui group, we identified mutations in phoP (S219L) and espK (I228M) genes. PhoP is part of two-component regulatory system $\mathrm{PhoP} / \mathrm{PhoQ}$. It is involved in transcriptional regulation of several virulence-associated genes and is also required for intracellular growth of M. tuberculosis (Pérez et al., 2001). Mutations in the phoP gene have been implicated in reduced survival and persistence of mycobacteria inside the host cell. PhoP controls the biosynthesis of sulfolipid (SL) and di- and polyacyltrehaloses (DAT and PAT) (Gonzalo Asensio et al., 2006; Walters et al., 2006). The mutation S219L in phoP gene affects the OmpR/PhoB-type DNA-binding which spans from 148 to 245 amino acids suggesting the role of this mutation in virulence attenuation. We identified the expression of phoP at both transcript and proteome level at higher abundance compared to the other proteins identified in this study.

Comparison of the In-Dels identified in our analysis with the structural variations reported by Elghraoui and Zheng et al., resulted in the identification of 14 insertions of which 8 led to frameshift mutations, 5 upstream variants and one inframe insertion (Supplementary Table 3B). We confirmed 9 deletions to be common among all studies, 3 of these being large deletions including $n r d H$-redoxin, IS6110 transposase and IS3 family transposase. NrdH is involved in electron transfer system for ribonucleotide reductase system. Phenotype profiling studies in M. tuberculosis has identified this protein to be essential for growth and cholesterol metabolism (Griffin et al., 2011). Transposable elements such as IS6110 can affect an organism's fitness through the insertional inactivation of genes (Yesilkaya et al., 2005). Carlos and groups have shown that IS6110 copy located within the promoter region of phoP affects the expression of several virulence-associated genes (Soto et al., 2004). Large deletions identified in IS6110 transposase need to be further investigated to study its role in virulence of $M$. tuberculosis. Among the genes identified with upstream deletion, we identified polyketide beta-ketoacyl synthase $p k s 3$, a gene well known to be involved in the cell wall lipid biosynthesis (Dubey et al., 2002).

\section{Protein Coding Evidence for Genes Supposed to Be "Absent" in H37Ra}

Our integrated analysis provides evidence of translation for 5 genes that were previously thought to be absent in H37Ra. Additionally, evidence at the transcript level was obtained for 9 genes inclusive of the above mentioned 5 genes. These 
include members of the esx 5 cluster namely es $x O$ and $e s x K$, as well as an enzyme required for arabinan synthesis (aftA) and a member of the universal stress family proteins. The findings from this study refute the reports published earlier with evidence provided at both transcript and proteome level. Further, a recently published study by our group could also quantify these proteins. Interestingly, the abundance of these apparently missing proteins did not vary significantly in both H37Rv and H37Ra strains (Table 1). For example, esxO recently shown to be involved in promoting intracellular persistence and host cell invasion upon ectopic expression in M. smegmatis (Mohanty et al., 2016), was expressed at similar levels in both log and stationary phase of H37Ra in comparison to H37Rv. A study on esxO also reports induction of autophagy and genomic instability in infected cells due to increase in host oxidative stress response. This suggests that there could be other mechanisms probably involving post-translation modifications that may likely play a role in modulating mycobacterial pathogenesis. Similarly, aft $A$ encoding arabinofuranosyltransferase and $R v 1184 c$ was expressed almost equally in both the strains. Interestingly, esxJ which was identified only at the transcript level in our study was expressed more that 2 -fold in the log phase of H37Ra in comparison to $\mathrm{H} 37 \mathrm{Rv}$.

\section{Proteomic Evidence for Putative Virulence Factors Identified in H37Ra}

Several investigations have been carried out for the identification of genes essential for mycobacterial virulence to understand the molecular basis of their virulence and persistence. Most of these genes encode for enzymes involved in lipid synthesis pathway, cell surface proteins encoding genes, regulators of metabolism and bacterial secretion system (Smith, 2003, Vander Beken et al., 2011). Genomic investigation of H37Ra from the current analysis revealed non-synonymous variations in six virulence-associated genes including membrane-associated phospholipase $\mathrm{C} 1$ and phospholipase C2 genes. Studies have shown that phospholipase C mutants in M. tuberculosis are attenuated in the late phase of the infection emphasizing the importance of phospholipases C in the virulence of the tubercle bacillus (Raynaud et al., 2002). Additionally, we provide evidence of expression of these genes at both transcript and proteome level, emphasizing expression of possibly non-functional phospholipases $\mathrm{C} 1$ and $\mathrm{C} 2$.

Further, comparative analysis of the putative virulence factors reported by Forrellad et al., with proteins identified uniquely in this study resulted in the identification of protein products of 12 putative virulence-associated genes. These proteins are known to be involved in the survival of mycobacterium inside the adverse microenvironment of host macrophage (Table 2) and include proteins belonging to the RNA polymerase sigma factor family namely, SigE and SigL, FAD-containing monoxygenase MymA, transporter proteins including mmpL8 among others. Interestingly, our study provides transcriptomic and proteomic evidence for all the seven genes belonging to the mymA operon. The operon in $M$. tuberculosis encode for proteins required for the proper ultrastructure of the cell envelope (Singh et al., 2005). Although $R v 3083$ or mymA has been identified in other mycobacterial species, our study provides the first proteomic evidence for its expression in M. tuberculosis H37Ra. This gene is known to have a role in modifying mycolic acid structure on the cell envelope of the mycobacteria thus helping them survive the acidic environment inside the macrophages (Saraav et al., 2017). The expression level of $m y m A$ in our data is comparatively lower than the other genes comprising this operon. It is therefore likely to have been missed in earlier studies owing to its low expression levels. Comparison with the expression profile from our previous study revealed that the first few genes of the mymA operon (Rv3084 and $R v 3085)$ were expressed at a lower level in H37Ra as compared to H37Rv (Verma et al., 2017). Additionally, Verma et al., have identified 35 putative virulence factors of which, 13 and 22 proteins were found to be upregulated and downregulated, respectively in $\mathrm{H} 37 \mathrm{Ra}$ with respect to $\mathrm{H} 37 \mathrm{Rv}$ (Fold change $\geq 2$ ). Among these, HspX was found to be more than two-fold downregulated in H37Ra stationary phase. This protein is known to possess chaperone activity with a significant ability to suppress the autolysis of $M$. tuberculosis and slow down the growth rate of bacteria during macrophage infection providing a survival advantage.

\section{Refinement of Genome Annotation Through Proteogenomics Analysis}

Using an integrated proteogenomic workflow, we report the identification of several novel peptides that enable correction of errors in genome assembly and annotation of gene models. Comparison of peptides corresponding to novel genes with peptides identified in Verma et al., revealed identification of 2 novel genes in H37Ra, which have been missed in the earlier genome annotation. Of this, one was found to be similar to PPE18 and the other to Rv1586c. Both these genes are expressed to a similar extent in both H37Ra and H37Rv. PPE18 is known to be required by the tuberculosis bacilli for its intracellular survival in the host and Rv1586c is known to play a role in integrating phiRv1, one among the 2 pro-phages common in the MTBC genomes, to its own genome (Fan et al., 2016). Thus, both of them are virulence associated factors in the virulent strain. Their identification in the avirulent strain further warrants the need for analysis that will ascertain the role of the currently known virulent factors.

Accurate annotation of the translational start sites (TSS) using gene prediction tools still remains a major challenge. It has been estimated that $60 \%$ of the assigned TSS are erroneous (Nielsen and Krogh, 2005). The genome of H37Ra shows high similarity with that of $\mathrm{H} 37 \mathrm{Rv}$ because they originated from a common parental strain (Jhingan et al., 2016). Approximately 60,33 , and $5 \%$ of the genes in M. tuberculosis are known to use ATG, GTG, and TTG as start codons, respectively (Kelkar et al., 2011). Although the initiator methionine is known to undergo formylation (fMet) in prokaryotes, N-terminal acetylation of proteins has also been observed in them, albeit at a lower rate compared to eukaryotes (Jones and O'Connor, 2011). Identification of these modified peptide sequences using mass 
spectrometry can be exploited to validate protein TSS (Prasad et al., 2017).

In silico studies have shown that bacterial genomes may comprise of undetected frameshift mutations (Elghraoui et al., 2017). Unrecognized frameshifts, stop codons in the frame and sequencing errors lead to interrupted coding sequences (ICDS) that can seriously affect the subsequent genome analysis. ICDS may be present in any gene of known or unknown function. For restoring the correct reading frame, several bacterial species have adopted the mechanisms of bypassing frameshifts. However, such incidences are rare. These sequences may be truly present in the organism and missed during sequencing or may arise from misannotation due to sequencing errors. One of the ICDSs identified in this study (NC_009525.1: 2,247,871-2,248,081), was found to be supported by 5 PSMs in proteomics analysis, where the sequence of known gene shifts open reading frames from frame 2 to frame 1 . The resulting protein sequence mapped to a hypothetical protein (WP_057368341.1) of H37Rv strain.

\section{CONCLUSIONS}

In this study, we successfully demonstrated the utility of multiomics approaches for the validation and correction of genomic data. Although a recent study suggested sequencing errors in the genome of H37Ra, no evidence was provided at the transcriptomic and proteomic levels. In the current study, we confirmed the identification of the genomic differences reported in previous studies. Furthermore, our study also provides evidence of the mutation spectrum in the promoter regions of certain virulence-associated genes. One of the important findings of this study include evidence of transcription and translation of genes that were previously reported as absent in H37Ra. These include genes that have been implicated in the mechanism of virulence and pathogen persistence in the host. Using an integrated proteogenomics pipeline, we also provide evidence of translation of several novel ORFs that have been annotated in other strains of $M$. tuberculosis including the virulent strain H37Rv but were missing in the genome annotation of H37Ra. The data generated from this study will pave the way for future studies on understanding the mechanism of virulence attenuation in H37Ra.

\section{AUTHOR CONTRIBUTIONS}

SP, SG, and TP contributed to conception and design of the study. SG provided the samples for the study. SP, RV, RR, and SK processed the samples. SP acquired mass spectrometry data. SP, RV, JA, OC, AP, SK, and YS analyzed the data. SP, JA, RV, OC, and

\section{REFERENCES}

Anders, S., Pyl, P. T., and Huber, W. (2015). HTSeq-a Python framework to work with high-throughput sequencing data. Bioinformatics 31, 166-169. doi: 10.1093/bioinformatics/btu638

Besemer, J., and Borodovsky, M. (2005). GeneMark: web software for gene finding in prokaryotes, eukaryotes and viruses. Nucleic Acids Res. 33, W451-W454. doi: $10.1093 /$ nar/gki487
AP prepared tables and figures. SP, RV, OC, SK, and YS carried out the literature search and wrote sections of the manuscript. All authors contributed to manuscript revision, read and approved the submitted version.

\section{ACKNOWLEDGMENTS}

The authors acknowledge Yenepoya University for access to mass spectrometry instrumentation facility. We also thank Karnataka Biotechnology and Information Technology Services (KBITS), Government of Karnataka for the support to the Center for Systems Biology and Molecular Medicine at Yenepoya University under the Biotechnology Skill Enhancement Programme in Multiomics Technology (BiSEP GO ITD 02 MDA 2017). SP is a recipient of INSPIRE Faculty Award from Department of Science and Technology (DST), Government of India. JA is a recipient of Senior Research Fellowship from Council of Scientific and Industrial Research (CSIR), Government of India. OC is a recipient of INSPIRE Fellowship from the Department of Science and Technology (DST), Government of India. SG is a Wellcome Trust/DBT India Alliance Intermediate Fellow.

\section{SUPPLEMENTARY MATERIAL}

The Supplementary Material for this article can be found online at: https://www.frontiersin.org/articles/10.3389/fmicb. 2018.01314/full\#supplementary-material

Supplementary Figure 1 | Representative MS/MS spectra of the proteins identified uniquely in our study and earlier reported to be absent in H37Ra.

Supplementary Table 1 | (A) List of mutations identified in H37Ra when data was aligned with H37Ra reference genome (Zheng et al.). (B) List of INDELS identified in H37Ra when data was aligned with H37Ra reference genome (Zheng et al.). (C) List of mutations identified in H37Ra when data was aligned with H37Ra reference genome (Elghraoui et al.). (D) List of INDELS identified in H37Ra when data was aligned with H37Ra reference genome (Elghraoui et al.).

Supplementary Table 2 | (A) List of mutations identified in H37Ra when data was aligned with H37Rv reference genome. (B) List of INDELS identified in H37Ra when data was aligned with H37Rv reference genome. (C) List of mutations in the promoter regions of 7 genes.

Supplementary Table 3 | (A) List of mutations common in our study, Zheng et al. and Elghraoui et al. (B) List of INDELS common in our study, Zheng et al. and Elghraoui et al.

Supplementary Table 4 | List of transcripts identified in this study.

Supplementary Table 5 | (A) List of proteins and peptides identified by LC-MS/MS analysis Mycobacterium tuberculosis H37Ra strain. (B) Comparative analysis of the list of proteins identified in previous published data and data from this study

Supplementary Table 6 | A summary of results from proteogenomics analysis. (A) Novel genes. (B) Protein extensions. (C) Alternate start site.

Bifani, P., Moghazeh, S., Shopsin, B., Driscoll, J., Ravikovitch, A., and Kreiswirth, B. N. (2000). Molecular characterization of Mycobacterium tuberculosis H37Rv/Ra variants: distinguishing the mycobacterial laboratory strain. J. Clin. Microbiol. 38, 3200-3204.

Braibant, M., Gilot, P., and Content, J. (2000). The ATP binding cassette (ABC) transport systems of Mycobacterium tuberculosis. FEMS Microbiol. Rev. 24, 449-467. doi: 10.1111/j.1574-6976.2000.tb0 0550.x 
Brosch, R., Philipp, W. J., Stavropoulos, E., Colston, M. J., Cole, S. T., and Gordon, S. V. (1999). Genomic analysis reveals variation between Mycobacterium tuberculosis $\mathrm{H} 37 \mathrm{Rv}$ and the attenuated M. tuberculosis H37Ra strain. Infect. Immun. 67, 5768-5774.

Chaerkady, R., Kelkar, D. S., Muthusamy, B., Kandasamy, K., Dwivedi, S. B., Sahasrabuddhe, N. A., Kim, M. S., et al. (2011). A proteogenomic analysis of Anopheles gambiae using high-resolution Fourier transform mass spectrometry. Genome Res. 21, 1872-1881. doi: 10.1101/gr.127951.111

Chesne-Seck, M. L., Barilone, N., Boudou, F., Gonzalo Asensio, J., Kolattukudy, P. E., Martin, C., et al. (2008). A point mutation in the two-component regulator PhoP-PhoR accounts for the absence of polyketide-derived acyltrehaloses but not that of phthiocerol dimycocerosates in Mycobacterium tuberculosis H37Ra. J. Bacteriol. 190, 1329-1334. doi: 10.1128/JB.01465-07

Collins, D. M. (2000). New tuberculosis vaccines based on attenuated strains of the Mycobacterium tuberculosis complex. Immunol. Cell Biol. 78, 342-348. doi: 10.1046/j.1440-1711.2000.00937.x

Cortes, T., Schubert, O. T., Rose, G., Arnvig, K. B., Comas, I., Aebersold, R., et al. (2013). Genome-wide mapping of transcriptional start sites defines an extensive leaderless transcriptome in Mycobacterium tuberculosis. Cell Rep. 5, 1121-1131. doi: 10.1016/j.celrep.2013.10.031

Dubey, V. S., Sirakova, T. D., and Kolattukudy, P. E. (2002). Disruption of msl3 abolishes the synthesis of mycolipanoic and mycolipenic acids required for polyacyltrehalose synthesis in Mycobacterium tuberculosis H37Rv and causes cell aggregation. Mol. Microbiol. 45, 1451-1459. doi: 10.1046/j.1365-2958.2002.03119.x

Elghraoui, A., Modlin, S. J., and Valafar, F. (2017). SMRT genome assembly corrects reference errors, resolving the genetic basis of virulence in Mycobacterium tuberculosis. BMC Genomics. 18:302. doi: 10.1186/s12864-017-3687-5

Fan, X., Abd Alla, A. A., and Xie, J. (2016). Distribution and function of prophage phiRv1 and phiRv2 among Mycobacterium tuberculosis complex. J. Biomol. Struct. Dyn. 34, 233-238. doi: 10.1080/07391102.2015.1022602

Forrellad, M. A., Klepp, L. I., Gioffre, A., Sabio y Garcia, J., Morbidoni, H. R., de la Paz Santangelo, M., et al. (2013). Virulence factors of the Mycobacterium tuberculosis complex. Virulence 4, 3-66. doi: 10.4161/viru.22329

Gonzalo Asensio, J., Maia, C., Ferrer, N. L., Barilone, N., Laval, F., Soto, C. Y., et al. (2006). The virulence-associated two-component PhoP-PhoR system controls the biosynthesis of polyketide-derived lipids in Mycobacterium tuberculosis. J. Biol. Chem. 281, 1313-1316. doi: 10.1074/jbc.C500388200

Griffin, J. E., Gawronski, J. D., Dejesus, M. A., Ioerger, T. R., Akerley, B. J., and Sassetti, C. M. (2011). High-resolution phenotypic profiling defines genes essential for mycobacterial growth and cholesterol catabolism. PLoS Pathog. 7:e1002251. doi: 10.1371/journal.ppat.1002251

He, X. Y., Zhuang, Y. H., Zhang, X. G., and Li, G. L. (2003). Comparative proteome analysis of culture supernatant proteins of Mycobacterium tuberculosis $\mathrm{H} 37 \mathrm{Rv}$ and H37Ra. Microbes Infect. 5, 851-856. doi: 10.1016/S1286-4579(03)00179-5

Jena, L., Kashikar, S., Kumar, S., and Harinath, B. C. (2013). Comparative proteomic analysis of Mycobacterium tuberculosis strain $\mathrm{H} 37 \mathrm{Rv}$ versus H37Ra. Int. J. Mycobacteriol. 2, 220-226. doi: 10.1016/j.ijmyco.2013.10.004

Jhingan, G. D., Kumari, S., Jamwal, S. V., Kalam, H., Arora, D., Jain, N., Kumaar, L. K., et al. (2016). Comparative proteomic analyses of avirulent, virulent, and clinical strains of Mycobacterium tuberculosis identify strain-specific patterns. J. Biol. Chem. 291, 14257-14273. doi: 10.1074/jbc.M115.666123

Jones, J. D., and O'Connor, C. D. (2011). Protein acetylation in prokaryotes. Proteomics 11, 3012-3022. doi: 10.1002/pmic.201000812

Kalscheuer, R., and Koliwer-Brandl, H. (2014). Genetics of mycobacterial trehalose metabolism. Microbiol Spectr. 2. doi: 10.1128/microbiolspec.MGM2-0002-2013

Karim, A. F., Chandra, P., Chopra, A., Siddiqui, Z., Bhaskar, A., Singh, A., et al. (2011). Express path analysis identifies a tyrosine kinase Src-centric network regulating divergent host responses to Mycobacterium tuberculosis infection. J. Biol. Chem. 286, 40307-40319. doi: 10.1074/jbc.M111.266239

Kelkar, D. S., Kumar, D., Kumar, P., Balakrishnan, L., Muthusamy, B., Yadav, A. K., et al. (2011). Proteogenomic analysis of Mycobacterium tuberculosis by high resolution mass spectrometry. Mol. Cell. Proteomics 10:M111.011627. doi: $10.1074 / \mathrm{mcp} . M 111.011445$

Li, H., and Durbin, R. (2009). Fast and accurate short read alignment with Burrows-Wheeler transform. Bioinformatics 25, 1754-1760. doi: 10.1093/bioinformatics/btp324
Målen, H., De Souza, G. A., Pathak, S., Softeland, T., and Wiker, H. G. (2011). Comparison of membrane proteins of Mycobacterium tuberculosis H37Rv and H37Ra strains. BMC Microbiol. 11:18. doi: 10.1186/1471-2180-11-18

McKenna, A., Hanna, M., Banks, E., Sivachenko, A., Cibulskis, K., Kernytsky, A., et al. (2010). The genome analysis toolkit: a mapreduce framework for analyzing next-generation DNA sequencing data. Genome Res. 20, 1297-1303. doi: $10.1101 /$ gr.107524.110

Mohanty, S., Dal Molin, M., Ganguli, G., Padhi, A., Jena, P., Selchow, P., et al. (2016). Mycobacterium tuberculosis EsxO (Rv2346c) promotes bacillary survival by inducing oxidative stress mediated genomic instability in macrophages. Tuberculosis 96, 44-57. doi: 10.1016/j.tube.2015.11.006

Mortazavi, A., Williams, B. A., McCue, K., Schaeffer, L., and Wold, B. (2008). Mapping and quantifying mammalian transcriptomes by RNA-Seq. Nat. Methods 5, 621-628. doi: 10.1038/nmeth.1226

Murphy, H. N., Stewart, G. R., Mischenko, V. V., Apt, A. S., Harris, R., McAlister, M. S., et al. (2005). The OtsAB pathway is essential for trehalose biosynthesis in Mycobacterium tuberculosis. J. Biol. Chem. 280, 14524-14529. doi: $10.1074 / j b c . M 414232200$

Nielsen, P., and Krogh, A. (2005). Large-scale prokaryotic gene prediction and comparison to genome annotation. Bioinformatics 15, 4322-4329. doi: 10.1093/bioinformatics/bti701

Pathan, M., Keerthikumar, S., Ang, C. S., Gangoda, L., Quek, C. Y., Williamson, N. A., et al. (2015). FunRich: an open access standalone functional enrichment and interaction network analysis tool. Proteomics 15, 2597-2601. doi: 10.1002/pmic.201400515

Pérez, E., Samper, S., Bordas, Y., Guilhot, C., Gicquel, B., and Martin, C. (2001). An essential role for phoP in Mycobacterium tuberculosis virulence. Mol. Microbiol. 41, 179-187. doi: 10.1046/j.1365-2958.2001.02500.x

Periwal, V., Patowary, A., Vellarikkal, S. K., Gupta, A., Singh, M., Mittal, A., et al. (2015). Comparative whole-genome analysis of clinical isolates reveals characteristic architecture of Mycobacterium tuberculosis pangenome. PLoS ONE 10:e0122979. doi: 10.1371/journal.pone.0122979

Philips, J. A., and Ernst, J. D. (2012). Tuberculosis pathogenesis and immunity. Annu. Rev. Pathol. 7, 353-384. doi: 10.1146/annurev-pathol-011811-132458

Potgieter, M. G., Nakedi, K. C., Ambler, J. M., Nel, A. J., Garnett, S., Soares, N. C., et al. (2016). Proteogenomic analysis of mycobacterium smegmatis using high resolution mass spectrometry. Front. Microbiol. 7:427. doi: $10.3389 /$ fmicb.2016.00427

Prasad, T. S., Mohanty, A. K., Kumar, M., Sreenivasamurthy, S. K., Dey, G., Nirujogi, R. S., et al. (2017). Integrating transcriptomic and proteomic data for accurate assembly and annotation of genomes. Genome Res. 27, 133-144. doi: $10.1101 /$ gr.201368.115

Raynaud, C., Guilhot, C., Rauzier, J., Bordat, Y., Pelicic, V., Manganelli, R., et al. (2002). Phospholipases C are involved in the virulence of Mycobacterium tuberculosis. Mol. Microbiol. 45, 203-217. doi: 10.1046/j.1365-2958.2002.03009.x

Renuse, S., Chaerkady, R., and Pandey, A. (2011). Proteogenomics. Proteomics. 11, 620-630. doi: 10.1002/pmic.201000615

Robinson, J. T., Thorvaldsdottir, H., Winckler, W., Guttman, M., Lander, E. S., Getz, G., et al. (2011). Integrative genomics viewer. Nat. Biotechnol. 29, 24-26. doi: 10.1038/nbt.1754

Sampson, S. L. (2011). Mycobacterial PE/PPE proteins at the host-pathogen interface. Clin. Dev. Immunol. 2011:497203. doi: 10.1155/2011/497203

Saraav, I., Singh, S., Pandey, K., Sharma, M., and Sharma, S. (2017). Mycobacterium tuberculosis MymA is a TLR2 agonist that activate macrophages and a TH1 response. Tuberculosis 106, 16-24. doi: 10.1016/j.tube.2017.05.005

Sassetti, C. M., Boyd, D. H., and Rubin, E. J. (2003). Genes required for mycobacterial growth defined by high density mutagenesis. Mol. Microbiol. 48, 77-84. doi: 10.1046/j.1365-2958.2003.03425.x

Schwanhäusser, B., Busse, D., Li, N., Dittmar, G., Schuchhardt, J., Wolf, J., et al. (2011). Global quantification of mammalian gene expression control. Nature 473, 337-342. doi: 10.1038/nature10098

Sharma, K., Verma, R., Advani, J., Chatterjee, O., Solanki, H. S., Sharma, A., et al. (2017). Whole genome sequencing of Mycobacterium tuberculosis isolates from extrapulmonary sites. OMICS 21, 413-425. doi: 10.1089/omi.2017.0070

Singh, A., Gupta, R., Vishwakarma, R. A., Narayanan, P. R., Paramasivan, C. N., Ramanathan, V. D., et al. (2005). Requirement of the mymA operon for appropriate cell wall ultrastructure and persistence of Mycobacterium 
tuberculosis in the spleens of guinea pigs. J. Bacteriol. 87, 4173-4186. doi: 10.1128/JB.187.12.4173-4186.2005

Smith, I. (2003). Mycobacterium tuberculosis pathogenesis and molecular determinants of virulence. Clin. Microbiol. Rev. 16, 463-496. doi: 10.1128/CMR.16.3.463-496.2003

Soto, C. Y., Menendez, M. C., Perez, E., Samper, S., Gomez, A. B., Garcia, M. J., et al. (2004). IS6110 mediates increased transcription of the phoP virulence gene in a multidrug-resistant clinical isolate responsible for tuberculosis outbreaks. J. Clin. Microbiol. 42, 212-219. doi: 10.1128/JCM.42.1.212-219.2004

Sreenivasamurthy, S. K., Madugundu, A. K., Patil, A. H., Dey, G., Mohanty, A. K., Kumar, M., et al. (2017). Mosquito-borne diseases and omics: tissue-restricted expression and alternative splicing revealed by transcriptome profiling of Anopheles stephensi. OMICS 21, 488-497. doi: 10.1089/omi.2017.0073

Vander Beken, S., Al Dulayymi, J. R., Naessens, T., Koza, G., Maza-Iglesias, M., Rowles, R., et al. (2011). Molecular structure of the Mycobacterium tuberculosis virulence factor, mycolic acid, determines the elicited inflammatory pattern. Eur. J. Immunol. 41, 450-460. doi: 10.1002/eji.201040719

Verma, R., Pinto, S. M., Patil, A. H., Advani, J., Subba, P., Kumar, M., et al. (2017). Quantitative proteomic and phosphoproteomic analysis of H37Ra and H37Rv strains of Mycobacterium tuberculosis. J. Proteome Res. 16, 1632-1645. doi: 10.1021/acs.jproteome.6b00983

Walters, S. B., Dubnau, E., Kolesnikova, I., Laval, F., Daffe, M., and Smith, I. (2006). The Mycobacterium tuberculosis PhoPR two-component system regulates genes essential for virulence and complex lipid biosynthesis. Mol. Microbiol. 60, 312-330. doi: 10.1111/j.1365-2958.2006.05102.x
Ye, K., Schulz, M. H., Long, Q., Apweiler, R., and Ning, Z. (2009). Pindel: a pattern growth approach to detect break points of large deletions and medium sized insertions from paired-end short reads. Bioinformatics 25, 2865-2871. doi: 10.1093/bioinformatics/btp394

Yesilkaya, H., Dale, J. W., Strachan, N. J., and Forbes, K. J. (2005). Natural transposon mutagenesis of clinical isolates of Mycobacterium tuberculosis how many genes does a pathogen need? J. Bacteriol. 87, 6726-6732. doi: 10.1128/JB.187.19.6726-6732.2005

Zheng, H., Lu, L., Wang, B., Pu, S., Zhang, X., Zhu, G., Shi, W., Zhang, L., Wang, H., Wang, S., et al. (2008). Genetic basis of virulence attenuation revealed by comparative genomic analysis of Mycobacterium tuberculosis strain H37Ra versus H37Rv. PLoS ONE 3:e2375. doi: 10.1371/journal.pone.00 02375

Conflict of Interest Statement: The authors declare that the research was conducted in the absence of any commercial or financial relationships that could be construed as a potential conflict of interest.

Copyright (c) 2018 Pinto, Verma, Advani, Chatterjee, Patil, Kapoor, Subbannayya, Raja, Gandotra and Prasad. This is an open-access article distributed under the terms of the Creative Commons Attribution License (CC BY). The use, distribution or reproduction in other forums is permitted, provided the original author(s) and the copyright owner are credited and that the original publication in this journal is cited, in accordance with accepted academic practice. No use, distribution or reproduction is permitted which does not comply with these terms. 